\title{
Cinq équipes de soins primaires face à la pandémie. Analyse des mobilisations territoriales
}

Five Primary care teams facing the Covid Pandemic: analysis of territorial mobilizations

François-Xavier Schweyer, Laure Fiquet, Sébastien Fleuret, Marie Blois, Marie Garnier, Antoine Géal et Aline Ramond-Roquin

\section{OpenEdition \\ Journals}

Édition électronique

URL : http://journals.openedition.org/rfst/976

DOI : $10.4000 /$ rfst.976

ISSN : 2492-3672

Éditeur

Espaces et SOciétés (UMR 6590)

Référence électronique

François-Xavier Schweyer, Laure Fiquet, Sébastien Fleuret, Marie Blois, Marie Garnier, Antoine Géal et Aline Ramond-Roquin, « Cinq équipes de soins primaires face à la pandémie. Analyse des mobilisations territoriales », Revue francophone sur la santé et les territoires [En ligne], Pandémie, crises et perspectives : lectures territoriales de la Covid-19, mis en ligne le 17 mars 2021, consulté le 06 avri 2021. URL : http://journals.openedition.org/rfst/976 ; DOI : https://doi.org/10.4000/rfst.976

Ce document a été généré automatiquement le 6 avril 2021.

\section{c) (i) (2)}

La Revue francophone sur la santé et les territoires est mise à disposition selon les termes de la Licence Creative Commons Attribution - Pas d'Utilisation Commerciale - Partage dans les Mêmes Conditions 4.0 International. 


\section{Cinq équipes de soins primaires face à la pandémie. Analyse des mobilisations territoriales}

Five Primary care teams facing the Covid Pandemic: analysis of territorial mobilizations

François-Xavier Schweyer, Laure Fiquet, Sébastien Fleuret, Marie Blois, Marie Garnier, Antoine Géal et Aline Ramond-Roquin

\section{Introduction}

1 Cet article propose une lecture territoriale de la pandémie de Covid-19 en analysant sa gestion par cinq équipes de soins primaires organisées en maisons de santé pluriprofessionnelles (MSP) dans deux régions, les Pays de la Loire et la Bretagne. Par équipe de soins primaires, on entend ici un regroupement de différents professionnels de santé libéraux et d'autres professionnels (secrétaire, coordinateur) autour d'un projet de santé commun déployé au service de la population locale dans le cadre d'une maison de santé pluri-professionnelle ${ }^{1}$ (MSP). Pour constituer une telle structure, au moins deux médecins généralistes et un paramédical doivent valider un projet de santé auprès de leur agence régionale de santé (ARS) (Fournier, 2014). Un contrat avec l'Assurance Maladie leur permet d'obtenir le financement de leurs activités pluri-professionnelles, si toutefois ils respectent des critères associés à trois grands objectifs : l'accès aux soins, la qualité et la coordination des soins, l'utilisation d'un système d'information partagé par l'équipe ${ }^{2}$.

2 L'étude présentée est issue d'un programme de recherche (Concert-MSP) ${ }^{3}$ en cours et qui porte sur la concertation entre d'une part, les professionnels de différents secteurs médical, médicosocial, social et, d'autre part, entre le «lieu MSP » et le reste du territoire (Ramond-Roquin et al, 2020). 
Cette concertation habituellement protéiforme et recouvrant un grand nombre de sujets a été chamboulée par la crise pour devenir quasi monothématique : un thème l'a emporté sur tous les autres, celui de la Covid-19. Notre positionnement a donc été d'observer les processus à l'œuvre dans cette reconfiguration de crise et notre choix a été d'adopter la perspective d'une analyse combinant deux dimensions territoriales: les territoires de proximité dans lesquels des équipes de MSP se sont adaptées à la situation inédite et les territoires régionaux qui ont été les espaces du déploiement des mesures de sécurité sanitaire décidées par l'Etat.

4 A la sortie du $1^{\mathrm{er}}$ confinement, trois internes en médecine générale effectuant un stage recherche ${ }^{4}$ ont été déployés sur le terrain de 5 des 10 MSP participant au programme Concert-MSP, choisies pour leur diversité en termes d'implantation, d'ancienneté, de nombre de professionnels de santé, pour collecter des données. Le premier interne couvrait les MSP 1 et 4 , le deuxième les MSP 2 et 5 situées en Pays de la Loire et le troisième la MSP 3 en région Bretagne. Leur mission a été enrichie d'une observation spécifique de l'impact de la crise Covid-19 sur les concertations pluri-professionnelles de juin à octobre 2020. L'enquête documentaire (compte-rendu des réunions durant la crise, protocoles mis en place, statistiques recueillies...), les observations participantes et les entretiens auprès de multiples acteurs (professionnels de santé, personnels de soutien [coordinatrices, secrétaires...] et partenaires des équipes de soins primaires) ont été analysés par l'équipe de recherche du projet Concert-MSP.

Il convient de souligner que la pandémie a été de faible intensité dans ces deux régions. Avec pour effet que les mesures conçues au niveau national ont mobilisé beaucoup d'acteurs sur les territoires, mais n'ont pas été systématiquement mises en œuvre, le taux d'incidence étant resté modéré.

Pour comprendre les enjeux sociaux et spatiaux des réponses apportées à la pandémie, on se propose d'étudier d'abord l'ancrage de ces équipes à leur territoire, qu'il s'agisse des contacts avec les acteurs locaux, de leur répertoire d'activité ou de l'implication de la population. Les géographes ont souligné l'imparfaite territorialisation de la santé au niveau local et le caractère protéiforme des réseaux territorialisés de première ligne en France (Fleuret, 2016). Si cette situation tend à évoluer suite à la loi HPST et à la mise en place de nombreux dispositifs territorialisés (GHT, CPTS, MSP, ESP-CLAP ${ }^{5} .$. ) le processus de territorialisation ne concerne pas encore la totalité du territoire français et recouvre des réalités et des définitions très variables selon les régions. Le territoire de santé est d'abord défini comme un espace d'organisation des soins et non par une approche globale (Coldefy, Lucas-Gabrielli, 2008). Nous nous demanderons si les équipes de soins primaires observées, dont l'exercice coordonné repose sur un projet de santé élaboré à partir d'un diagnostic territorial, conduisent à nuancer ces constats.

Ces équipes s'inscrivent par ailleurs dans des écologies différentes (Schweyer, Vezinat, 2019). Plusieurs échelles d'organisation s'emboîtent: l'échelon régional pour la définition et le financement des politiques de santé, l'échelon départemental où sont regroupés un certain nombre d'acteurs en santé, l'échelon des territoires de santé ou " pays » dans lesquels évoluent les équipes de soins primaires et l'échelon communal ou intercommunal (EPCI) voire même pour l'une des MSP du panel l'échelon infracommunal (quartier politique de la ville). Les modalités de gestion de cette crise avant, pendant et après le premier confinement (mars-juillet 2020) ont traduit des politiques territoriales différenciées. Il s'agira d'analyser le rôle des agences régionales de santé, 
les différents acteurs mobilisés, les formes d'intégration des MSP au système de santé régional ainsi que dans leur environnement local.

8 Après avoir présenté l'ancrage territorial de cinq équipes de soins primaires (1), nous analyserons comment s'est déployée l'organisation de la gestion de crise sur les territoires, sous l'influence de quels acteurs et à quelle échelle (2). Nous étudierons ensuite comment les équipes ont adapté leurs pratiques et quelles formes de solidarité se sont manifestées (3) avant de nous demander pour conclure si la gestion de la pandémie a modifié l'articulation santé territoire.

\section{L'ancrage territorial et organisationnel des équipes de soins primaires}

9 Les cinq équipes considérées ici évoluent dans différents types de territoires (2 régions, 4 départements). Deux façons de classifier nous ont parues pertinentes et ont été adoptées : la première selon le type de territoire desservi, la seconde selon la logique spatio-organisationnelle, qui traduit les priorités que se donnent les équipes pour répondre aux besoins des territoires.

Dans le cadre du programme de recherche Concert-MSP, une typologie sommaire a été élaborée à partir des données collectées pour l'ensemble des MSP du programme. Cette typologie n'était pas une finalité de recherche mais visait à distinguer les MSP selon des éléments de similarité ou de différenciation des territoires dans lesquels elles sont implantées. Il s'agissait de pouvoir analyser les variations observées dans les modalités de concertation d'une catégorie territoriale à une autre pour évaluer l'importance du facteur " territoire ». Par conséquent, il fallait se baser sur des indicateurs disponibles à l'échelle locale et comparables d'une région à une autre. Nous avons donc procédé à une collecte d'indicateurs territorialisés (indicateurs socio-sanitaires, démographiques, socioéconomiques, etc.) à partir des sources suivantes : données de l'INSEE, données des observatoires régionaux de santé et portraits de territoires que la CPAM fournit pour chaque MSP. Ces indicateurs sont très rarement disponibles à la même échelle et l'échelle de disponibilité varie selon les régions. Il a donc été décidé de travailler au plus petit dénominateur commun soit l'échelle des EPCI (Etablissements publics de coopération intercommunale). Cette échelle permet de récolter un nombre limité, insatisfaisant à bien des égards, mais suffisant d'indicateurs tout en conservant une échelle fine du territoire.

11 Les indicateurs choisis sont classés en trois catégories (offre de soins, population, structure spatiale) en référence à la typologie publiée par Chevillard et Mousquès (2018). Le choix a été fait de ne pas reprendre cette typologie telle quelle, car celle-ci vise d'autres objectifs : une classification nationale des territoires de vie. Notre volonté était différente. Il s'agissait de se placer à l'échelle des territoires desservis par les MSP (qui ne correspondent pas aux territoires de vie) et de choisir des données disponibles à cette échelle. Nous ne souhaitions pas situer les MSP dans une typologie nationale, mais classifier spatialement notre échantillon, ce qui relève d'une démarche différente. Néanmoins la classification en 3 catégories fournissait une base de travail sur laquelle 
nous nous sommes appuyés pour sélectionner les données qui ont permis de classifier les territoires des MSP :

- offre de soins et de services (accessibilité potentielle localisée des médecins généralistes, densité d'infirmier.e.s en libéral, densité de sages-femmes, places en établissement d'hébergement pour personnes âgées dépendantes (EHPAD), nombre d'affections longue durée-ALD) ;

- Population (ratio cadres/ouvriers, part du logement social, part des familles monoparentales, part de propriétaires, taux de chômage, indice de vieillissement, taux de mortalité, part des bénéficiaires de la couverture maladie universelle complémentaire);

- Structure spatiale (rural/urbain, Environnement politique des MSP -parti politique majoritaire, taux de participation aux élections municipales de 2020 / moyenne EPCI-, existence ou non d'un contrat local de santé, nombre de sites composant la MSP).

Le caractère disparate de ces données ainsi que les possibles redondances statistiques de certaines interdisaient un traitement statistique multivarié, la typologie a donc été réalisée selon la méthode de la matrice ordonnable développée par Bertin (1970 et 1980) et a fait apparaitre trois types de territoires :

- Type 1 (T1): les territoires à tendance rurale des MSP 1, 2 et 3 se distinguent par une part moyenne de la population bénéficiant de l'ALD, une faible capacité d'accueil en EHPA/ EHPAD, une faible part de logements sociaux dans le parc résidentiel et une part importante de propriétaires.

- Type 2 (T2) : le territoire rural intermédiaire de la MSP 5 se distingue par une densité moyenne de médecins, une importante population bénéficiaire de l'ALD, une forte part de la population propriétaire, peu de familles monoparentales et un fort taux de participation aux élections municipales.

- Type 3 (T3) : le territoire à tendance urbaine de la MSP 4 se distingue par une forte densité de médecins, une forte part de logements sociaux dans le parc résidentiel, une forte part de familles monoparentales et un fort taux de chômage.

13 Au-delà des caractéristiques propres aux territoires, les équipes considérées ici présentent aussi une variabilité en termes d'inscription territoriale. On entend par là d'une part, une combinaison de facteurs territoriaux plus ou moins favorables au projet d'exercice coordonné. Parmi eux, citons la démographie médicale, la mobilisation d'acteurs locaux sur les questions de santé ou les liens existant ou non avec les autorités sanitaires. L'inscription territoriale traduit aussi, d'autre part, la logique spatio-organisationnelle adoptée par chaque équipe. Il est possible de distinguer trois logiques territoriales parmi les cinq équipes étudiées :

- Logique spatio-organisationnelle S0 1 « logique du maintien local » : l'équipe de la MSP 5 est de petite taille, dans un secteur rural et moyennement dense en offre de soins. Le regroupement des professionnels vise en premier lieu à maintenir une offre de soins en proximité immédiate, plutôt qu'à déployer des projets à large échelle en s'associant avec les professionnels des territoires adjacents. Le soutien des élus locaux a été déterminant pour le maintien de la MSP, alors qu'à l'échelle régionale, la tutelle interrogeait la pertinence de ce projet de petite envergure.

- Logique spatio-organisationnelle SO 2 «logique de fonctionnement centrée sur les professionnels »: les équipes des MSP 2 et 3 se sont développées autour de liens locaux affinitaires. Dans ces équipes, les professionnels se sont au cours du temps, positionnés collectivement pour proposer des réponses aux différentes problématiques auxquelles ils avaient à faire face. Dans la MSP 2, les professionnels se sont d'abord regroupés pour mieux 
répondre aux demandes de soins programmés et non programmés, mais aussi pour proposer des actions de prévention et promotion de la santé, et élargir l'offre de soins de proximité, par exemple dans le champ de la périnatalité, de la santé mentale ou de la nutrition. La MSP 3 a permis de maintenir une offre de soins de proximité, de développer un accès à des consultations spécialisées et de proposer des actions d'éducation thérapeutiques. Des liens forts avec les autres MSP situés à proximité ont été développés par la création d'une association, préfiguration d'une $\mathrm{CPTS}^{6}$.

- Logique spatio-organisationnelle SO 3 « logique populationnelle »: les équipes des MSP 1 et 4 ont en commun de rassembler un grand nombre d'acteurs issus de différents secteurs, dans une perspective d'offre de santé intégrée et de dimension populationnelle. Pour la MSP 1 , il s'agit d'un modèle d'intégration rurale, en situation de quasi-monopole : tous les acteurs de santé du territoire ou presque sont réunis autour d'un projet commun et y contribuent, et la population de ce territoire bien défini s'adresse de manière quasi exclusive à ce groupe d'acteurs. Ce pôle de santé regroupe les secteurs ambulatoire et hospitalier de proximité ainsi que le secteur médico-social, en partenariat direct avec l'ARS. Pour la MSP 4, située en quartier prioritaire de la ville, il s'agit d'une intégration plus locale sur un territoire plus difficile à circonscrire, que les différents acteurs ne partagent pas forcément, et qui évolue avec le temps (rénovation du quartier, mobilité des habitants et des services...). Cette équipe a développé des partenariats avec de nombreux interlocuteurs aux logiques différentes, incluant des professionnels libéraux, des professionnels salariés d'associations, mais aussi des institutions publiques et des élus locaux. Il existe un investissement important de cette équipe dans des actions de santé communautaire, d'éducation à la santé dont le champ d'action déborde largement les seules patientèles des professionnels.

Enfin, les cinq équipes étudiées ici présentent aussi une variabilité sur plusieurs éléments dont l'effet pourrait avoir été sensible sur l'organisation d'une réponse à la crise de la Covid-19. On peut retenir d'une part, le fait qu'une MSP soit mono site ou multi sites (voir tableau) et, d'autre part, l'étendue du territoire desservi, avec des distances à parcourir pour accéder aux soins et aux services ou pour les dispenser.

Tableau 1 : Caractéristiques générales des 5 équipes de soins primaires

\begin{tabular}{|c|c|c|c|c|c|c|c|c|}
\hline & $\begin{array}{c}\text { Date } \\
\text { projet } \\
\text { de } \\
\text { santé }\end{array}$ & Région & Département & $\begin{array}{c}\text { Nombre de } \\
\text { professionnels }\end{array}$ & $\begin{array}{c}\text { Nombre } \\
\text { de } \\
\text { patients } \\
\text { ayant un } \\
\text { médecin } \\
\text { traitant } \\
\text { dans la } \\
\text { structure }\end{array}$ & Structure & $\begin{array}{c}\text { Typologie } \\
\text { territoriale }\end{array}$ & $\begin{array}{c}\text { Typologie } \\
\text { Orga }^{\text {elle }}\end{array}$ \\
\hline $\begin{array}{c}\text { MSP } \\
1\end{array}$ & 2009 & $\begin{array}{l}\text { Pays de } \\
\text { La Loire }\end{array}$ & Mayenne & $>40$ & $\begin{array}{l}15000- \\
20000\end{array}$ & $\begin{array}{l}\text { Multi- } \\
\text { site }\end{array}$ & $\mathrm{T} 1$ & SO 3 \\
\hline $\begin{array}{c}\text { MSP } \\
2\end{array}$ & 2012 & $\begin{array}{l}\text { Pays de } \\
\text { La Loire }\end{array}$ & Sarthe & $10-20$ & $\begin{array}{c}5000 \\
-10000\end{array}$ & $\begin{array}{l}\text { Mono- } \\
\text { site }\end{array}$ & T1 & SO 2 \\
\hline $\begin{array}{c}\text { MSP } \\
3\end{array}$ & 2013 & Bretagne & $\begin{array}{l}\text { Ille et } \\
\text { Vilaine }\end{array}$ & $>40$ & $\begin{array}{c}5000 \\
-10000\end{array}$ & $\begin{array}{l}\text { Multi- } \\
\text { site }\end{array}$ & $\mathrm{T} 1$ & SO 2 \\
\hline $\begin{array}{c}\text { MSP } \\
4\end{array}$ & 2013 & $\begin{array}{l}\text { Pays de } \\
\text { La Loire }\end{array}$ & $\begin{array}{c}\text { Maine et } \\
\text { Loire }\end{array}$ & $10-20$ & $<5000$ & $\begin{array}{l}\text { Mono- } \\
\text { site }\end{array}$ & T3 & SO 3 \\
\hline
\end{tabular}




\begin{tabular}{|c|c|c|c|c|c|c|c|c|}
\hline $\begin{array}{c}\text { MSP } \\
5\end{array}$ & 2014 & $\begin{array}{c}\text { Pays de } \\
\text { La Loire }\end{array}$ & $\begin{array}{c}\text { Maine et } \\
\text { Loire }\end{array}$ & $10-20$ & $<5000$ & $\begin{array}{c}\text { Mono- } \\
\text { site }\end{array}$ & T2 & SO 1 \\
\hline
\end{tabular}

Lecture : la typologie territoriale distingue les territoires à tendance rurale (T1), rural intermédiaire (T2), à tendance urbaine (T3). Les logiques spatio-organisationnelles se répartissent en trois types : maintien local (SO1), centré sur les professionnels (SO2) et populationnel (SO3)

\section{L'espace d'organisation de la gestion de crise}

En ce qui concerne la gestion de la crise en soins primaires, la politique nationale a été mise en œuvre dans des configurations régionales différentes et déployée selon des approches départementales spécifiques, dépendant des jeux d'acteurs en présence, et des déclinaisons territoriales particulières.

\section{Le plan ORSAN déployé dans deux régions}

En France, la gestion épidémique repose sur le dispositif ORSAN REB (Organisation de la Réponse du système de santé en situations Sanitaires exceptionnelles risque épidémiologique ou biologique), mis en œuvre par les ARS. Il vise une réponse graduée en trois stades afin d'organiser l'endiguement de la pathologie et assurer un accès aux soins de la population. Dans le cadre de la crise Covid-19, son déploiement a été progressif. Le stade 1 déclenché le 23 février 2020 (Ministère de la Santé, 2019) a concerné 38 établissements ayant pour mission d'organiser les soins des patients suspects d'infection et ainsi d'en limiter la propagation. Le stade 2, décrété le 28 février, a impliqué les établissements de santé disposant de plans blancs dans le cadre d'une stratégie d'endiguement, visant à freiner l'introduction et la propagation du virus sur le territoire national, qui officiellement n'était pas en circulation active (Bergeron et al, 2020). Pendant ces deux premières phases, les professionnels de soins primaires n'étaient pas mobilisés, le plan blanc ne concernant que les établissements de santé (Bourgueil et al, 2020).

17 Le passage en stade 3 à partir du 14 mars, appelé "stade épidémique ", a modifié la stratégie d'intervention (stratégie collective d'atténuation, visant à endiguer les effets de la circulation active du virus) et a conduit à dépasser la gestion de la crise au sein des seuls établissements de santé. L'ensemble des composantes du système de santé : établissements de santé, soins ambulatoires (« soins de ville ») et secteur médico-social, a été mobilisé. Ce changement de stratégie visait à assurer la prise en charge des patients sans signe de gravité en ambulatoire et à réserver les ressources hospitalières aux seuls patients présentant des signes de gravité, en phase de circulation active du virus où il existait un risque de saturation du système hospitalier.

En ce qui concerne la régulation des cas suspects d'infection Covid-19, alors qu'initialement ces patients étaient d'abord tous orientés vers le centre 15, ils ont été incités à partir de la mi-mars à contacter en priorité leur médecin traitant. Le centre 15 restait le recours pour ceux présentant des symptômes graves (ou ceux n'ayant pas de médecin traitant), afin d'éviter la saturation des plateformes de régulation.

Pour la prise en charge des patients suspects d'infection Covid-19, la déclinaison des filières de soins a été différente dans les deux régions étudiées. Schématiquement, l'ARS des Pays de la Loire a privilégié une approche où les patients suspects d'infection 
Covid-19 étaient dirigés vers des lieux dédiés, séparés des lieux de soins habituels, pour

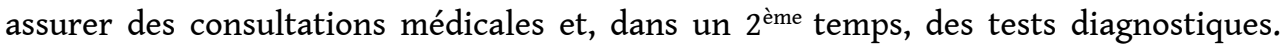
L'ARS Bretagne a quant à elle déployé une approche basée sur les organisations existantes après les avoir répertoriées, et effectué un travail de suivi.

\section{Les MSP face aux déclinaisons départementales du plan en Pays de la Loire}

Le choix régional de l'ARS Pays de la Loire visait à mutualiser les ressources, en termes d'équipements de protection individuelle, à sécuriser les patients en tentant de séparer les flux de patients suspects de Covid-19 de ceux considérés non suspects, et, dans certains secteurs, à assurer l'accès aux soins pour les patients suspects de Covid-19 sans médecin traitant.

Dans les différents départements de cette région, les centres hospitaliers (CHU, GHT), l'ADOPS ${ }^{7}$, les Conseils de l'ordre départementaux et certaines équipes de MSP ont organisé une réponse basée sur ces lieux dédiés, dans des maisons médicales de gardes, dans des locaux non destinés aux soins habituellement (salles municipales) ou parfois dans des locaux non utilisés de certaines MSP. Les patients y étaient adressés par les secrétariats des cabinets de médecine générale, ou parfois par la régulation téléphonique du 15 et du $116117^{8}$.

Dans le département des MSP 4 et 5 (Maine et Loire), 28 centres dédiés Covid-19 ont émergé dès le 19 mars, sous l'impulsion du $\mathrm{CHU}$ et de médecins généralistes volontaires. Chaque médecin coordonnateur de centre pouvait s'organiser de façon autonome pour les plannings, la répartition des tâches entre professionnels, le système d'informations et surtout le lien avec les partenaires locaux Les médecins généralistes étaient incités à adresser aux centres dédiés tout patient suspect de Covid-19, les tutelles leur rappelant le risque sanitaire majeur à prendre en charge des patients suspects d'infection au sein de leurs propres cabinets. Cette organisation a été fortement soutenue par le $\mathrm{GHT}^{9}$ départemental, le Conseil départemental, le $\mathrm{CDOM}^{10}$ et la Faculté de santé, cette dernière mettant à disposition des ressources humaines (étudiants en médecine) et du matériel (fabrication de gel hydro-alcoolique par le Département de pharmacie).

3 Les équipes de soins primaires du département n'ont pas été impliquées en tant que telles dans ce dispositif. Les médecins de la MSP 5 ont participé au dispositif en réalisant des consultations dans le centre dédié de proximité et en y orientant les patients suspects de Covid-19. Cette MSP, située à la limite du département, couvre un territoire sur 3 départements (de la même région). Si l'équipe entretient des liens avec de multiples acteurs de santé, les médecins n'ont participé qu'au dispositif du département sur lequel la MSP est implantée.

Dans la MSP 4, la plupart des médecins ont également consulté dans le centre dédié sans s'impliquer davantage. Plusieurs limites ont conduit les professionnels à ne plus $y$ adresser systématiquement les patients suspects. Implantée en quartier populaire, avec une large proportion de patients précaires, souvent allophones, l'équipe a dû en effet adapter quasi individuellement les messages et les actions pour les rendre accessibles aux habitants. L'accès aux soins était compliqué pour les plus précaires dans les centres. La méconnaissance des problématiques des patients aux besoins complexes et les difficultés de transmission de l'information vers l'équipe habituelle du patient ont conduit les professionnels à préférer prodiguer les soins eux-mêmes. Avec le temps, des 
pratiques multiples ont progressivement cohabité, certaines s'appuyant sur les centres dédiés, et d'autres permettant, grâce à des adaptations internes, l'accueil des patients suspects Covid-19 par des membres de l'équipe.

Cette organisation départementale du Maine et Loire a été mise en place sans tenir compte des organisations de soins primaires, suscitant des tensions dans sa mise en œuvre et dans sa gouvernance.

Dans le département de la MSP 1 (Mayenne), 8 centres dédiés Covid-19 ont été mis en place dès le 23 mars. L'ADOPS en a été le principal acteur ainsi que du suivi. La PTA (Plateforme territoriale d'appui) ${ }^{11}$ était chargée d'assurer un accès aux soins pour les patients infectés Covid-19 sans médecin traitant. L'ADOPS collectait chaque jour les données d'activité de chaque centre, permettant de mettre en évidence une chute d'activité progressive, avant une hausse fin juin. Ces centres ont fermé leurs portes le 10 juillet suite à l'arrêt, vivement critiqué, du soutien financier de l'ARS, dans un contexte de rebond épidémique mais de fréquentation des centres restant relativement faible.

27 L'équipe de la MSP 1, entretenant un lien ancien et fort avec les autorités sanitaires, s'est articulée avec le dispositif départemental, tout en revendiquant et mettant en place une organisation spécifique. Sous l'impulsion d'une "cellule de crise COVID " rassemblant très régulièrement différents professionnels de l'équipe, les médecins ont organisé dès le 16 mars un circuit et des créneaux de consultations (puis de tests) dédiés aux patients suspects de Covid-19. La régulation des appels reposait directement sur le secrétariat médical de l'équipe. L'équipe de cette MSP rurale a fait le choix d'assurer une offre de soins de proximité en accueillant les patients suspects COVID sur 3 lieux de consultations. Pour les deux sites principaux de la MSP, dans des locaux de l'équipe (alors inoccupés par certains professionnels ayant dû suspendre leur activité), ce qui assurait un accès simple à la population et des conditions de pratiques habituelles pour les professionnels. Le $3^{\text {ème }}$ site de la MSP, plus petit, avait sollicité le prêt d'un local par la mairie pour accueillir ces consultations dédiées. Le partenariat existant entre la MSP et l'hôpital local (lui-même intégré au GHT ${ }^{12}$ départemental), a été mis à profit dans la gestion de crise, (mutualisation du matériel, établissement de protocoles pour la prise en charge des patients suspects ou encore organisation des parcours de soins ville-hôpital). L'ARS a reconnu ce fonctionnement en assurant un soutien financier identique à celui accordé aux autres centres.

L'organisation départementale de la Mayenne s'est ainsi déployée en centres dédiés à partir des médecins généralistes impliqués dans l'ADOPS tout en permettant aux acteurs d'adapter l'organisation localement (MSP1).

Dans le département de la MSP 2 (Sarthe), 11 centres dédiés se sont progressivement déployés : 6 centres en collaboration avec le service départemental de santé au travail, 4 MSP (hors du programme Concert-MSP) et un centre de prévention de la CPAM. Disposant d'un soutien logistique et financier de l'ARS, ces centres ont accueilli les patients suspects de Covid-19, en particulier ceux présentant des facteurs de risque de formes graves et/ou ceux n'ayant pas de médecin traitant. Ils ont également assuré la réalisation de tests diagnostiques en complément de l'activité des laboratoires d'analyse médicale, et le suivi téléphonique des patients sans médecin traitant testés COVID +.

L'équipe de la MSP 2 s'est rapidement mobilisée pour être en mesure d'orienter et de prendre en charge elle-même sur son territoire les patients suspects de Covid-19, sans 
recourir aux centres départementaux dédiés, hormis pour la réalisation des tests diagnostiques. Ainsi, un protocole d'orientation téléphonique et d'accueil physique des patients a été établi et régulièrement mis à jour L'équipe a eu peu d'échanges avec l'ARS, la CPAM, le CDOM, ou le centre hospitalier de recours, et a ressenti une compréhension limitée des besoins des soins primaires dans le dispositif départemental " officiel ». L'équipe a sollicité la reconnaissance et le soutien financier de l'ARS au même titre que les équipes de soins primaires officiellement « labellisées » par l'Agence mais sa taille et ses moyens humains relativement limités et l'absence de réalisation des tests diagnostiques, ne le permettaient pas initialement. Néanmoins, l'ARS a attribué a posteriori une enveloppe budgétaire à l'équipe, qui l'a perçue comme une reconnaissance de sa mobilisation, sans être à la hauteur des attendus.

31 Sur ce département de la Sarthe, certaines équipes de soins primaires se sont affranchies de l'organisation en centres pour continuer d'accueillir leurs patients au sein de la MSP, sans avoir de lien majeur avec l'ARS.

\section{L'approche « réaliste » de la région Bretagne}

En Bretagne, l'approche a été qualifiée de "réaliste» par l'ARS qui a soutenu les organisations des acteurs locaux, en les répertoriant et en suivant leur activité quotidienne (Deborne, 2020). Les messages diffusés autour de l'organisation des soins de ville ont mis en avant une organisation centrée sur le « médecin traitant » et pointé les différentes organisations déployées (ARS Bretagne, 2020). Les professionnels de santé étaient déjà organisés localement lorsque l'ARS les a sollicités. Dans le département de la MSP 3 (Ille et Vilaine), une demande a été envoyée à l'ensemble des cabinets médicaux pour répertorier les organisations locales et savoir si les structures de soins étaient en capacité d'accueillir des patients suspects de Covid-19 y compris lorsqu'ils n'avaient pas de médecin traitant.

La stratégie régionale a cherché à répertorier l'offre de la médecine de ville en créant une carte interactive à destination des professionnels de santé (médecins traitants, SAMU, centre 15). Il s'agissait d'orienter les patients vers les structures existantes et d'identifier les territoires sans organisation spécifique ou n'ayant pas souhaité être recensés par l'ARS. Le choix a été fait de se baser sur une organisation par Pays, échelon territorial d'action spécifique à cette région (Deborne, 2020), après une concertation avec les acteurs des soins de ville : URPS médecins, ADOPS, et certains professionnels de MSP.

34 Concrètement, deux types de structures ont coexisté. D'une part, des sites de soins organisés par les professionnels pour faire face aux demandes de soins de patients Covid-19 dans des lieux de consultation déjà existants (MSP, cabinets de groupes). Dans ces structures, un circuit spécifique aux patients suspects de Covid-19 ou présentant des symptômes infectieux a été mis en place (créneaux de consultations dédiés ou locaux séparés). Et d'autre part, dans certains territoires, des centres ambulatoires Covid-19 réservés aux patients suspects d'infection ont également été créés, pour proposer des consultations dans des locaux le plus souvent communaux (salles des fêtes, gymnases) par des professionnels de santé locaux préférant externaliser les consultations hors de leur cabinet pour différentes raisons (absence de locaux adaptés, volonté de ne pas exposer des médecins plus âgés, protocoles d'hygiène renforcés organisés). Sur quelques territoires, il s'agissait d'un second niveau d'organisation en cas de surcharge de consultations dans les sites de soins organisés, pour pouvoir faire 
face à une possible augmentation de patients atteints de COVID. Le faible niveau de l'épidémie explique que localement ces structures aient été finalement laissées en sommeil. Le lien entre l'ARS et les sites de soins organisés ou les centres ambulatoires ad hoc a été constant, en vue de répertorier chaque semaine l'activité: nombre de patients atteints, nombre de consultations, nombre de patients hospitalisés, etc.

La MSP 3 s'est ainsi organisée dès la mi-mars pour proposer des locaux dédiés aux consultations de patients suspects d'infection Covid-19. La régulation des appels était assurée par le secrétariat médical de la structure à l'aide de documents fournis par l'ARS ou par l'URPS médecins. La MSP étant multisite, un des bureaux situés sur le site où le plus grand nombre de médecins généralistes exerçaient, a été dédié aux patients suspects, avec un circuit spécifique de circulation (un patient suspect ne devait pas croiser un autre patient). Les médecins se relayaient pour accueillir les patients avec une volonté de préserver certains professionnels considérés à risque (femmes enceintes ou professionnels âgés). Cette MSP a été contactée par l'ARS après la mise en place de cette organisation et a été répertoriée comme une des 180 structures pouvant recevoir des patients suspects de COVID y compris des patients qui n'avaient pas de médecin traitant. Le centre 15 avait la liste des structures recevant ces patients et pouvait donc les adresser spécifiquement sur cette MSP. La possibilité d'ouvrir un deuxième bureau a été envisagée mais n'a pas été nécessaire. Les horaires de ces consultations dédiées ont varié en fonction des besoins. Un centre exclusivement dédié COVID a été pensé dans une commune située à 20 kilomètres, mais n'a pas été mis en fonctionnement puisque les différents cabinets de groupes ou MSP du secteur ont réussi à recevoir l'ensemble des patients suspects ou non durant cette période.

Tableau 2 : Déclinaisons locales et territoriales de la gestion de la crise

\begin{tabular}{|c|c|c|c|c|c|}
\hline $\begin{array}{c}\text { Département / } \\
\text { territoire }\end{array}$ & Structure & Organisation & Acteurs & Activités & Évolution \\
\hline $\begin{array}{c}\text { Maine et Loire } \\
\text { T2 }\end{array}$ & $\begin{array}{c}\text { MSP 5/ } \\
\text { SO1 }\end{array}$ & $\begin{array}{c}\text { Centre Covid } \\
\text { (CC) externe }\end{array}$ & $\begin{array}{c}\text { Simple } \\
\text { participation CC } \\
\text { extérieur }\end{array}$ & $\begin{array}{c}\text { Simple } \\
\text { participation CC } \\
\text { extérieur }\end{array}$ \\
\hline $\begin{array}{c}\text { Maine et Loire } \\
\text { T3 }\end{array}$ & $\begin{array}{c}\text { MSP 4 } \\
\text { SO3 }\end{array}$ & $\begin{array}{c}\text { Centre Covid } \\
\text { externe }\end{array}$ & $\begin{array}{c}\text { CHU, GHT, } \\
\text { Cniv, Cdom, } \\
\text { cl Depart }\end{array}$ & $\begin{array}{c}\text { Simple } \\
\text { participation CC } \\
\text { extérieur }\end{array}$ & $\begin{array}{c}\text { Progressif accueil } \\
\text { local patients } \\
\text { Covid+ }\end{array}$ \\
\hline $\begin{array}{c}\text { Mayenne T1 } \\
\text { Sarthe T1 }\end{array}$ & $\begin{array}{c}\text { MSP 2 / } \\
\text { SO2 }\end{array}$ & $\begin{array}{c}\text { Centre Covid } \\
\text { dans MSP }\end{array}$ & $\begin{array}{c}\text { ADOPS, PTA, } \\
\text { Samu, Cdom }\end{array}$ & $\begin{array}{c}\text { Centre Covid } \\
\text { dans MSP }\end{array}$ & $\begin{array}{c}\text { Réception directe } \\
\text { appels }\end{array}$ \\
\hline Ille et Vilaine T2 & $\begin{array}{c}\text { MSP 3 / } \\
\text { SO2 }\end{array}$ & Locaux dédiés \\
en interne & ARS, Centre & 15 & $\begin{array}{c}\text { Accueil suspects } \\
\text { Covid + }\end{array}$ & $\begin{array}{c}\text { Continuité et } \\
\text { labellisation ARS }\end{array}$ \\
\hline
\end{tabular}

Lecture : la typologie territoriale distingue les territoires à tendance rurale (T1), rural intermédiaire (T2), à tendance urbaine (T3). Les logiques spatio-organisationnelles se répartissent en trois types : maintien local (SO1), centré sur les professionnels (SO2) et populationnel (SO3). Le sens des acronymes utilisés est : CHU pour Centre Hospitalo-Universitaire, GHT pour Groupement hospitalier de territoire, Univ pour université, Cdom pour Conseil départemental de l'Ordre des médecins, PTA pour plateforme territoriale d'appui, Cel Dal pour Conseil départemental. 
différentes approches régionales et territoriales ont eu des conséquences sur l'implication des professionnels de santé dans la gestion de la crise, mais aussi sur la perception de leur rôle face aux autorités sanitaires et sur l'adaptation de leurs organisations de travail.

\section{Le territoire comme espace des liens sociaux : pratiques, solidarités, tensions}

La gestion territoriale de la crise a mis en lumière différentes définitions de la notion de territoire. En tant que maillage fin de l'organisation du plan ORSAN, le territoire d'action publique locale est celui des pouvoirs publics, des élus, des collectivités territoriales en partenariat avec les services sociaux et médicosociaux, notamment de l'aide à domicile. Il s'agit aussi d'un bassin de vie où une population tisse des relations d'échange, des relations de voisinage dans un espace géographique qui structure les déplacements quotidiens. L'analyse des activités des professionnels de santé dans le cadre des MSP mobilise une nouvelle définition de la notion de territoire, de nature sociologique. Il s'agit du territoire professionnel, au sens de juridiction (Abbott, 1988). Chaque groupe professionnel travaille à définir un territoire d'intervention spécifique délimité par des frontières qui font parfois obstacle à la coopération (Schweyer, 2004). La crise a bousculé ces territoires en renouvelant la question de la prise en charge des patients. Au sein de chaque équipe s'est posée une double question: qui prend en charge quoi? Avec qui ? L'urgence sanitaire a conduit à mobiliser des réseaux de relations entre pairs, à instaurer des alliances, à permettre des délégations et des coopérations entre professionnels de santé et avec d'autres métiers dont le rôle a été mis en lumière. Mais les juridictions ne dépendent pas que des seules professions, elles sont négociées avec l'Etat, comme l'a rappelé la gestion de la crise où certaines professions ont été empêchées d'exercer en cabinet libéral (orthophonistes, diététiciennes, podologues, kinésithérapeutes, etc.)

La pandémie a conduit les équipes à modifier leurs pratiques de soins et de coopération en mettant en place de nouveaux modes d'organisation, parfois de nouveaux rôles et de nouvelles pratiques dont la pérennité pose aujourd'hui question. Ces transformations ont été, non sans difficultés, l'occasion d'initiatives et de solidarités professionnelles. Des réseaux d'entraide locaux ont déplacé les frontières instituées du système sanitaire.

\section{L'adaptation des organisations et des pratiques}

Dans toutes les MSP, une nouvelle répartition des rôles professionnels s'est imposée. Le plan ORSAN a instauré une " première ligne de soins » animée par quelques professions tandis que les autres professionnels étaient tenus en retrait.

\section{La première ligne et les empêchés diversement mobilisés}

Trois professions de santé ont été en première ligne car jugées indispensables à la gestion de la crise : les médecins, les infirmières et les pharmaciens. Pour beaucoup, les médecins ont alterné consultations COVID (en réalisant parfois des tests PCR) et consultations classiques avec, au début du confinement, une baisse de la fréquentation des cabinets constatée. Les médecins ont fait face à un important afflux quotidien de 
mails des différentes institutions : "DGS URGENT », CPAM, ARS, Conseil de l'Ordre ou encore URPS. Les directives ciblées visaient une multitude de thématiques, des stratégies de dépistage aux dispositions funéraires.

Les infirmières ont gardé une activité importante, essentiellement à domicile, avec un certain stress ressenti face au risque de contamination dû au manque d'équipements de protection. Pour beaucoup, elles ont cessé d'assurer leurs permanences au sein des MSP, tout en y étant actives pour contribuer à la réorganisation des locaux et des activités.

Les pharmaciens ont vu leurs missions se modifier avec un contact plus rapproché des patients (en particulier avec les renouvellements d'ordonnances possibles sans consultation médicale) et un rôle de conseil majoré, certains usagers craignant de se rendre au cabinet médical. L'activité a été soutenue surtout au début du confinement avec des liens forts avec les autres professionnels actifs, une implication majeure dans la distribution des équipements de protection individuels, mais aussi dans l'organisation des consultations COVID.

43 Les secrétaires ont également joué un rôle important avec une charge de travail augmentée. Là où les coordinatrices avaient un temps de présence suffisant, elles ont contribué à organiser le travail de "cellules de crise ", à accompagner les adaptations récurrentes de pratiques et d'organisation de l'équipe, ainsi qu'à diffuser des informations auprès des autres professionnels et à maintenir les liens. Cette implication, particulièrement chronophage, a souvent nécessité de suspendre leurs activités habituelles.

Les autres professions "empêchées » d'exercer en cabinet ont en général stoppé leur activité pendant la période de confinement. Pour ceux qui avaient une activité mixte ville-hôpital (MSP 1) ils ont augmenté le temps en structure. Les kinésithérapeutes ont vécu un retrait forcé de leur cabinet sur avis du conseil de l'Ordre, avec la consigne de maintenir les soins urgents à domicile (dont la définition a parfois fait l'objet de d'échange au sein des équipes). Certains ont appelé des patients pour leur prodiguer des conseils et indiqué des vidéos sur Youtube. Certains professionnels salariés (infirmière de santé publique) ou agents d'accueil de la mairie (MSP 4) ont cessé leur activité au sein de la MSP à la demande de leur employeur.

\section{Déplacements de rôles et nouveaux acteurs}

Le déplacement des rôles professionnels a été particulièrement visible en ce qui concerne les secrétaires. De nouvelles missions sont apparues générant parfois du stress (changements d'organisation répétés, fiches COVID à remplir, appels des patients, activité de régulation à assurer). Dans la MSP 5, la secrétaire chargée de l'orientation des patients devait leur poser des questions pour en décider et a parfois dû affronter leur attitude quand, prenant mal que ce soit la secrétaire « qui joue le rôle du médecin ", ils refusaient de l'écouter. Elle a vécu comme "difficile» de faire appliquer les gestes barrières, de devoir «faire la morale aux gens». Ailleurs, des professionnels de santé ont épaulé les secrétaires sans que la solidarité ne soit toujours efficace. L'aide dans la prise des appels tout confondu (COVID ou non) a été tempérée par le manque de connaissance des logiciels.

Les renouvellements d'ordonnances n'ont pas été l'apanage des seuls pharmaciens, les infirmières les ont également assurés en lien avec le médecin traitant. 
47 Les pharmaciens quant à eux ont eu des nouvelles missions par l'Etat comme le repérage des violences conjugales. Si un affichage a été mis en place en pharmacie (devenue un point relai), ils n'ont pas eu de situation à gérer (MSP 2).

Le travail réalisé par des personnels des caisses d'assurance maladie en charge d'appeler les patients testés + est peu mentionné si ce n'est sur le territoire de la MSP 3, où l'intervention des "brigades de la sécurité sociale» a suscité des réactions contrastées au sein de l'équipe: un des médecins trouvait ce système peu efficace tandis qu'une infirmière exposait son efficacité pour repérer et isoler un groupe de patients atteints.

\section{La réorganisation des activités}

L'activité quotidienne des équipes a été substantiellement modifiée par la priorité donnée à la gestion de l'épidémie. Les activités spécifiquement organisées au sein de MSP comme la concertation pluri-professionnelle ont été délaissées ou adaptées au contexte de crise. Seuls les patients en grande fragilité en ont bénéficié dans la MSP 1 où les réunions mensuelles du pôle ont également été stoppées jusqu'en septembre. Dans plusieurs MSP, le confinement a obligé à décaler les consultations de spécialistes, avec parfois une grande difficulté pour gérer ces changements. Les professionnels restés sur place, particulièrement les médecins, ont été en revanche beaucoup plus mobilisés par des réunions formalisées. Des médecins ont fait part d'échanges d'informations, de protocoles entre confrères, en particuliers via les réseaux sociaux. Les interactions avec d'autres MSP voisines, régionales et même nationales ont semblé plus fréquentes.

50 L'usage de la télémédecine, mis en place par l'ensemble des équipes, n'est toutefois pas apparu dans les MSP étudiées comme un élément structurant du travail en temps de pandémie ${ }^{13}$. Il a été discuté dans certaines MSP sans faire l'unanimité, et a été utilisé de façon très variable selon les équipes et les professionnels. Lors des réunions COVID de la MSP 3, la place des téléconsultations a été discutée sans aboutir à un consensus. Un des médecins, favorable à la réalisation de téléconsultations pour adresser directement une partie des patients vers les tests PCR n'a pas été suivi par ses confrères qui jugeaient que cette pratique pouvait faire passer à côté d'un autre diagnostic.

\section{Les effets de la COVID sur la dynamique collective}

51 La mobilisation des équipes face à la pandémie n'a pas eu les mêmes effets partout. Dans certaines MSP, le modèle relationnel est resté stable mais s'est comme intensifié avec une concertation accrue. Si le fort leadership médical existant dans la MSP 1 a persisté et s'est même accentué durant la période, le fonctionnement de la MSP n'a pas changé. Il a été toutefois vécu plus intensément avec « sur le moment un sentiment de se rapprocher", une "grande émulation" dans un investissement collectif pour permettre une prise en charge sécurisée des patients : « Le COVID aura permis de mieux s'exprimer, de mieux travailler ensemble ». Cette perception, rapportée par la plupart des professionnels, notamment les plus investis au printemps (médecins, infirmières, pharmaciens), n'a toutefois pas été partagée par l'ensemble de l'équipe. Certains paramédicaux contraints à interrompre leur activité ont au contraire ressenti une certaine mise à l'écart durant la période initiale du confinement. Au sein de la MSP 4, le «noyau dur » composé des médecins et de la coordinatrice, très actif pour gérer la 
crise, existait auparavant et sans que n'émerge de leadership médical. Un rapprochement entre l'association des IDE et les médecins généralistes a été observé, avec des échanges informels sur le temps de midi et le développement assez symbolique d'un système de commande de repas groupée. Le lien entre les professionnels durant la période a été maintenu par la coordinatrice qui a assuré la cohésion entre les différents membres de l'équipe, y compris en restant en relation avec les paramédicaux restés chez eux par la création d'un groupe WhatsApp.

\section{Renforcement des liens, solidarité et tensions}

Dans plusieurs MSP, la mobilisation face à la crise a resserré les liens. Les pharmaciens ont souvent eu de multiples échanges avec les membres de leurs équipes. Certains paramédicaux, bien qu'en retrait d'activité, ont pu apprécier l'entraide et le dialogue avec d'autres professionnels. Une orthophoniste par exemple a félicité les secrétaires et trouvé rassurant d'être en MSP, bénéficiant d'informations pour la réorganisation de son activité. Dans la MSP 3 multi-site, la crise a été l'occasion pour des IDE ayant leur cabinet dans un site éloigné de venir à la MSP « centre », de se rapprocher de l'équipe, de mieux connaître leurs collègues, de vivre plus sereinement la situation, ravies "d'avoir rejoint la MSP ». Au sein de cette même MSP3, une nouvelle activité de concertation s'est mise en place toutes les semaines, sur le temps de la pause déjeuner, multipliant par quatre la fréquence de concertation entre professionnels favorisant l'émergence d'une dynamique particulière et d'une plus grande motivation à travailler ensemble. La crise a mis en évidence de manière concrète l'intérêt de la concertation et de l'exercice pluri-professionnel pour mieux s'organiser. Un médecin reconnaît avoir été «beaucoup plus régulier " aux réunions qu'à son habitude et que " tout le monde s'était senti obligé de s'impliquer ». Au sein de centres dédiés, le travail avec d'autres médecins libéraux retrouvés dans les permanences a été vécu comme positif et créateur de liens.

Toutefois, le vécu de la crise n'a pas toujours été aisé. Le surtravail, le stress, l'incertitude ont été éprouvants. Il a été fait appel à la psychologue pour assister les membres de l'équipe dans une MSP ou dans certaines pharmacies où l'enjeu de la protection au sein de l'équipe avait généré une « angoisse importante ». Le fait qu'une équipe exerce sur différents lieux géographiques a pu complexifier le travail d'articulation entre les acteurs et a nécessité des ajustements réguliers; la crise a mis au jour des fonctionnements différents selon les lieux, avec des divergences de prise en charge selon les sites (différences de critères d'orientation vers une consultation COVID). Dans ces cas, tout se passe comme si l'homogénéité des équipes s'était construite sur des activités et des outils stabilisés et que le niveau élevé d'incertitude introduit par la pandémie avait fait resurgir des pratiques et des modes d'exercice différents. Le travail en commun déployé au sein des équipes n'a pas empêché l'expression de divergences entre professionnels sur l'application des mesures d'hygiène ou la compréhension des protocoles. Si cette période a «révélé » de belles relations, elle a aussi mis en lumière "la personnalité de certains" sans toutefois menacer le « travailler ensemble».

Le retrait de certains professionnels n'a pas été sans conséquences. Pour les professionnels de la MSP 1 ayant cessé leur activité durant la période, les relations lors du retour ont pu être compliquées, avec le ressenti d'un manque de communication de la part des médecins et l'impression d'être mis à l'écart des décisions. Ailleurs, c'est le 
désinvestissement de professionnels en arrêt qui n'a pas été compris. La maturité des équipes semble avoir joué le rôle d'antidote à la fragilisation des relations interpersonnelles. Les professionnels de la MSP 4 n'ayant pas poursuivi leur activité sur place pendant le confinement ont été conviés à certaines des réunions de crise mais n'y ont pas participé. A leur retour après le confinement, ils n'ont pas exprimé de sentiment d'abandon mais au contraire, une confiance, avec l'assurance que les mesures mises en place par les autres professionnels facilitaient leur reprise.

\section{Le territoire de proximité comme référence et ressource} d'atteinte » qui, par peur, manque de moyens de transports, ont cessé de consulter. La situation était identique dans le quartier populaire de la MSP4 où, dès sa reprise d'activité, l'infirmière de santé publique (salariée de la ville) a pris l'initiative d'organiser des maraudes pour "aller vers" les habitants et ainsi mieux comprendre leurs besoins et leurs attentes. Le message adressé initialement aux patients de ne consulter que pour les soins d'urgence a eu un effet négatif pour certains et on peut faire l'hypothèse que la décision d'indemniser des consultations "bilan et vigilance " $(46 €)$ pour une période prolongée au-delà du confinement a été un moyen politique de compenser cet effet pervers. quartier populaire de la MSP 4, les téléconsultations ont rapidement été compliquées auprès d'une patientèle précaire en partie sans matériel informatique et peu à l'aise avec les outils. 
59 Les liens avec le secteur psychiatrique souvent compliqués, ont été aggravés lors de la période COVID, avec la fermeture de certains lieux d'accueil hospitaliers ainsi que des CMP, dans une période de forte nécessité vis-à-vis de patients fragilisés par la crise.

\section{Les conséquences de la crise sur les logiques d'organisation territoriale}

60 La crise sanitaire semble avoir questionné les logiques spatio-organisationnelles présentées en partie 1.

\section{Logique spatio-organisationnelle 1 : la logique du maintien}

61 Pour la MSP 5, cette logique semble s'être poursuivie, avec néanmoins un agrandissement du périmètre territorial. Un médecin s'est mis en relation avec un confrère d'une MSP voisine afin de travailler sur l'organisation à adopter dans ce contexte COVID. Ce partenariat était d'autant plus novateur qu'un projet de fusion des deux MSP avait avorté auparavant et il a permis d'améliorer les représentations mutuelles entre équipes. Des rôles professionnels ont évolué avec l'élargissement du rôle de la secrétaire.

\section{Logique spatio-organisationnelle 2 : la logique professionnelle}

Il semble que, pour ces deux MSP (2 et 3), le territoire de la MSP se soit élargi, avec pour la MSP 3 une progressive orientation vers une logique plus populationnelle.

La MSP 2 s'est affranchie du fonctionnement départemental pour proposer un accès aux soins des patients suspects de COVID pour organiser une réponse de proximité. L'implication des élus locaux semble avoir été renforcée (prêt de voiture et service civique).

La MSP 3 semble avoir étendu son territoire et intégré de nouveaux acteurs locaux (nouveaux cabinets d'infirmiers). Des réunions en visioconférence hebdomadaires des infirmières ont été organisées avec le soutien et la collaboration de la coordinatrice. Des groupes de messageries en ligne ont été créés pour faciliter les échanges entre infirmières, entre coordinatrices des MSP du territoire ou encore entre médecins du département. Des critiques ont été exprimées contre certains laboratoires locaux quand l'insuffisance de créneaux de prélèvements par tests PCR a généré des délais de prise de rendez-vous et des résultats tardifs obligeant des médecins à adresser certains patients vers les établissements hospitaliers de la ville voisine. L'accès aux soins des patients de la MSP3 a ainsi été questionné permettant d'interpeller des acteurs locaux sur leur fonctionnement ou de repérer les patients ne consultant plus. Des échanges amorcés au sein de l'association des MSP sur le territoire préfigurant la CPTS se sont intensifiés. Des psychologues ont organisé des permanences téléphoniques gratuites relayées par la $\mathrm{PTA}^{8}$ pour apporter un soutien psychologique à la population. Une logique d'offre de soins à la population semble ainsi avoir été à l'œuvre, sans doute favorisée par l'autonomie laissée aux structures pour s'organiser en région Bretagne. 


\section{Logique spatio-organisationnelle 3 : La logique populationnelle} l'offre au niveau local. Quelles seront les consequences au long cours de la sanitaire sur les liens de l'équipe avec ses partenaires habituels locaux? L'arrêt de toutes les actions communautaires pendant la période du confinement et l'arrêt de l'accueil de patients précaires par les structures associatives a multiplié les « perdus de vue » et a détérioré les conditions de possibilité d'accès aux soins. Alors que la MSP 4 avait l'habitude de beaucoup travailler en lien avec la mairie, le quartier et l'ensemble des structures locales, la période a été marquée par un décalage important entre l'équipe et ses partenaires municipaux. La fermeture de lieux gérés par la mairie, ainsi que le retrait des salariés hors de la MSP ont été vécus comme la confirmation de constats antérieurs sur des modes de fonctionnement et des points de vue différents. L'activité des structures sociales et de prévention (PMI, médecine scolaire...) a cessé sur certains territoires.

\section{Un retour à la normale avec quelques acquis}

68 Au moment de l'enquête (automne 2020), une reprise des activités de consultations habituelles était constatée avec en parallèle une organisation COVID différente selon les MSP. Globalement, les organisations dédiées créées au printemps (centres COVID départementaux des Pays de la Loire) n'étaient plus actives, et la prise en charge des patients suspects d'infection Covid-19 (ou confirmés) se poursuivait de façon plus intégrée aux pratiques habituelles. Le sentiment de s'être rapproché s'est atténué avec la reprise de la routine. Le dispositif COVID initialement bien utilisé a progressivement été délaissé pour une gestion plus individuelle de patients spécifiques dont les médecins préféraient assurer eux-mêmes le suivi. Le retour en présentiel des paramédicaux marque la fin assez nette de cette période un peu particulière avec la reprise des activités habituelles (réunions cliniques, pluri-professionnelles). Dans la MSP 4, le rapprochement qui s'était effectué entre le Centre de soins infirmiers et les médecins n'aura finalement été que temporaire.

\section{Discussion}

L'analyse présentée est centrée sur première vague de la pandémie. Après le déconfinement, la pandémie a connu un autre pic qui n'a pas bousculé autant la vie des 
équipes, mieux préparées et moins exposées à l'hospitalo-centrisme de la première réponse sanitaire.

Les cinq MSP étudiées ici se sont adaptées pour faire face à cette crise sanitaire en modifiant leurs pratiques. Cette dynamique d'adaptation ne semble pas spécifique à ces structures, elle a été également observée au niveau national. L'enquête du réseau ACCORD (2020) a décrit au sein des MSP et centres de santé étudiés des procédures d'accueil spécifiques des patients COVID (dans 95\% des structures), un renforcement de la coopération avec les partenaires locaux (80\%), la mise en place d'une cellule de crise (67\%), moins souvent des procédures spécifiques de suivi des patients non COVID (34\%). L'intérêt de notre étude est de comprendre ces adaptations au prisme de la territorialisation, au sens que lui donnent Eliot, Lucas-Gabrielli et Mangeney (2017), c'est-à-dire comme un processus à l'interface de la gouvernance (coopération), du gouvernement (délimitation de l'action) et des pratiques (usages). Ce processus favorisant le jeu entre les échelles spatiales et organisationnelles et les «coopérations souples » sous forme de partenariats volontaires, peu formalisés et flexibles.

L'apparition de l'épidémie a provoqué une réaffirmation soudaine de l'autorité de l'Etat, garant de la sécurité sanitaire et qui, en l'espèce, n'a pas seulement gouverné à distance mais aussi de près (Dolez, Donier, 2020). La crise a été un moment de verticalité où les ARS ont été les transmetteurs des instructions ministérielles vis-à-vis des professionnels de santé hospitaliers et libéraux, avec toutefois pour ces derniers une relation plus directe à l'échelon départemental (Pribile, 2020) et un effet de différenciation territoriale dû aux éléments d'horizontalité générés par les mobilisations locales.

Comme nous l'avons montré dans la deuxième partie, le rôle des ARS, en charge de l'application du plan ORSAN REB, a été central et visait à adapter la pertinence de l'action publique aux spécificités territoriales. La légitimité du plan national et sa contribution à l'intérêt général n'ont pas été questionnés dans leur principe. En revanche, dans certains départements, la légitimité du leadership départemental ( $\mathrm{CHU}$, CDOM par exemple) a fait l'objet de tensions et de controverses locales. Les territoires en tant qu'espaces d'application du plan national se sont différenciés selon le niveau de prise en compte de la mobilisation des acteurs locaux et les nécessaires compromis à passer pour assoir l'autorité de l'Etat (Bourgueil et al, 2020). Deux approches différentes ont caractérisé les régions étudiées, définies en fonction des acteurs et des modes de collaboration déjà en place dans une logique de pathdependence (Pierson, 1993). Avec pour conséquence une différenciation des formes de mise en œuvre du plan, en partie imposée par les professionnels de terrain qui entendaient faire reconnaitre leurs actions et initiatives pour anticiper ou modifier le plan officiel. Si l'ARS de la région Bretagne a intégré les initiatives professionnelles locales dans l'application régionale du plan, dans les Pays de la Loire, la situation a été plus contrastée selon les territoires. Dans le département du Maine et Loire, des initiatives des autorités se sont imposées aux acteurs qui les ont plus ou moins contournées ou contestées (MSP4). Dans les départements de la Mayenne et de la Sarthe, certaines initiatives locales se sont imposées aux autorités. On a vu par exemple que la MSP 1 et la MSP 2 ont demandé, et obtenu, des financements tout en ayant agi en dehors des critères du cahier des charges officiel.

73 Il convient de rappeler que les deux régions concernées par l'analyse ont été relativement épargnées par l'épidémie. Tous les éléments prévus dans le dispositif de 
gestion de crise n'ont pas été déployés et les équipes mobilisées pour l'accueil des patients infectés ont été modérément exposées. Cette moindre pression a sans doute favorisé un espace d'initiatives locales pour répondre à la crise. Toutefois, la différenciation territoriale observée dans la gestion de la crise s'explique pour une bonne part dans la configuration du territoire ressource de chaque MSP. Par exemple, la MSP 1 adossée à un hôpital de proximité a pu gérer la crise par elle-même, ce qui était impossible pour la MSP 4 à la fois proche d'un CHU «chef de file » et dont la population servie est difficile à amener vers le soin. La MSP 3 loin d'un hôpital et en relation avec une délégation départementale ARS encourageant les initiatives locales a pu gérer de façon assez autonome le dispositif Covid.

74 La crise a par ailleurs contribué à redéfinir le territoire de certaines MSP quand la mise entre parenthèse des routines professionnelles et le confinement, ont eu pour effet d'ouvrir considérablement l'espace des échanges, bien au-delà du territoire d'exercice. Le manque de maîtrise de l'épidémie a entraîné un questionnement permanent, avec une recherche d'informations auprès des sources officielles, mais aussi auprès des autres professionnels du territoire. La participation conjointe à un Centre COVID a rapproché les professionnels d'un territoire (MSP 5, 3), la mise à disposition d'une liste avec des coordonnées à jour a rendu les liens plus faciles avec les spécialistes, y compris du CHU, par ailleurs plus accessibles (MSP 4, 3). On a également observé l'émergence ou le renforcement de réseaux d'entraide locaux dépassant les frontières instituées du système sanitaire. La gestion de la pandémie par les équipes des MSP a-t-elle pour autant modifié localement l'articulation santé territoire? Les manifestations de solidarité locale concernant les équipements de protection individuelle et le respect des consignes comportementales ont mobilisé les élus, les habitants, les entreprises. En ce sens, la lutte contre l'épidémie a été l'affaire de tous. Mais les conséquences psychologiques et sanitaires, notamment pour les populations fragiles, restent aujourd'hui à évaluer. Il est probable que la conception du plan ORSAN qui n'a mobilisé les soins primaires qu'en stade 3 sera interrogée à l'avenir.

La pandémie a conduit les équipes des MSP étudiées à modifier leurs pratiques de soins et de coopération et à concevoir de nouveaux modes d'organisation. La gestion de la crise, en priorisant l'offre de soins dédiés à la Covid-19, a mis en retrait les soins courants et l'approche globale qui est celle des soins primaires. Or permettre l'accès aux soins en proximité est une valeur partagée et mobilisatrice pour beaucoup de professionnels de santé. De ce point de vue, la crise a, au moins dans un premier temps, plutôt accru la faible territorialisation de la santé au niveau local, bien que les réseaux professionnels locaux se soient souvent renforcés, au moins en ce qui concerne les trois professions placées en première ligne, à savoir les médecins, les infirmières et les pharmaciens. Les transformations observées n'ont pas été sans difficultés bien qu'elles aient révélé des capacités d'initiatives et des solidarités professionnelles. En ce sens, la crise COVID a été une épreuve de professionnalité en exercice coordonné. Au point de vue de la coopération intersectorielle, le confinement a eu pour effet de diviser deux mondes, celui des soignants restés actifs au contact des patients et celui des salariés des services sociaux et médico-sociaux en arrêt ou télétravail. Au sein des équipes de soins primaires, la crise a interrogé les territoires professionnels, la division symbolique du travail et a pu contribuer à donner de nouveaux rôles et à instaurer de nouvelles pratiques. Des questions restées longtemps sous-jacentes ont été posées et réfléchies en commun, et certaines décisions prises semblent pérennes. Par exemple, des médecins qui assurent à tour de rôle une plage dédiée au COVID selon un planning de 
consultation et un emploi du temps type, ou une centralisation des résultats COVID via le secrétariat qui permet une lecture suivie, ou un secrétariat renforcé, des permanences infirmières regroupées ou remplacées par des rendez-vous, ou encore, de façon plus informelle, la mise en place de repas partagés. On observe également une forme d'apprentissage du travail en réunion, avec un nombre accru de participants. Toutefois, si l'enquête a permis de documenter une intensification de la coopération entre professionnels, elle ne permet pas de dire que la nécessité des rôles complémentaires ait effacé les hiérarchies symboliques. La coopération entre professions semble avoir été favorisée et justifiée par la crise sans conduire cependant à interroger les statuts réciproques, l'urgence était ailleurs.

Enfin, peut-on dire qu'une réponse adaptée aux besoins a été possible là où existait un système d'action local, c'est-à-dire là où les professionnels se connaissaient à travers un réseau de relations plus ou moins formalisé ou un exercice coordonné ? Il apparaît que l'existence d'un collectif de travail a été facilitant pour élaborer des réponses appropriées. L'importance du rôle joué par les métiers de service (secrétaires, coordinatrices) a été mise en lumière lors des opérations de réorganisation des locaux et du travail au sein des MSP. De même que l'apport d'une solidarité d'équipe pour faire face aux aléas. Le rôle central des médecins a, lui, été plutôt renforcé. L'incertitude générée par la pandémie a suscité des contacts interprofessionnels à l'échelon des territoires, au-delà des seules équipes constituées. Cet élargissement des réseaux relationnels et l'interconnaissance entre acteurs des territoires qu'il a permis, pourraient compter parmi les acquis de la crise.

\section{Conclusion}

77 L'analyse de la réponse apportée à la pandémie de Covid 19 par cinq équipes de MSP montre des mobilisations territoriales et des pratiques différenciées. Dans chaque cas, plusieurs échelles territoriales s'articulent (plan de sécurité sanitaire et règles professionnelles définis au niveau national, pilotage des ARS déployé au niveau régional et adapté selon les départements, professionnels de santé mobilisés au niveau local avec les acteurs des territoires de proximité), mais la combinaison de ces échelles et leurs influences sont variables au niveau des MSP. La différenciation des pratiques observées s'explique en partie par les spécificités des territoires (analysées en trois types) et les logiques spatio-organisationnelles des MSP, mais aussi par l'articulation entre le dispositif de sécurité sanitaire et ses instruments, la coopération entre les acteurs professionnels et des territoires et les pratiques professionnelles issues d'initiatives locales et de logiques d'appropriation diverses. En ce sens, la crise Covid 19 met en lumière les effets du processus de territorialisation dans le secteur des soins primaires. 


\section{BIBLIOGRAPHIE}

ABBOTT A.,1988, The System of Professions. An Essay on the Division of Expert Labor, University of Chicago, Chicago Press.

ARS Bretagne, 2020, [en ligne] https://www.bretagne.ars.sante.fr/system/files/2020-04/CPCOVID19_medecine-de-ville_030420.pdf Consulté le 13 décembre 2020

ARS Pays de la Loire, 2020, [en ligne] https://www.pays-de-la-loire.ars.sante.fr/Covid-19-la-priseen-charge-et-le-suivi-des-patients-atteints-par-le-coronavirus-dont-letat-de Consulté le 13 décembre 2020

BERGERON H., BORRAZ O., CASTEL P., DEDIEU F.,2020, Covid-19: une crise organisationnelle, Paris, Sciences Po Les Presses.

BERTIN J., 1970, La graphique, Communications, 15(1), 169-185.

BERTIN J., 1980, Traitements graphiques et mathématiques. Différence fondamentale et complémentarité. Mathématiques et sciences humaines, 72, 60-71.

BOURGUEIL Y, FALCOFF H, RAMOND-ROQUIN A, SAVIGNEAU G, BOUCHEZ T., 2020, La première vague de Covid-19 en France et les soins primaires, Rev Med Suisse 2020 ; volume 16. 2123-2126

COLDEFY M., LUCAS-GABRIELLI V., 2008, Les territoires de santé: des approches régionales variées de ce nouvel espace de planification, Irdes, document de travail $\mathrm{n}^{\circ} 10$.

CHEVILLARD G., MOUSQUES J., 2018, « Accessibilité aux soins et attractivité territoriale : proposition d'une typologie des territoires de vie français », Cybergeo: European Journal of Geography [En ligne], Espace, Société, Territoire, document 873, mis en ligne le 21 novembre 2018, consulté le 03 décembre 2020. URL : http://journals.openedition.org/cybergeo/29737 ; DOI : https://doi.org/10.4000/cybergeo.29737

DEBORNE M., 2020, Gérer la crise Covid-19 dans les territoires de proximité, Mémoire de Master 2 Mention Santé publique, EHESP.

DOLEZ B., DONIER V., 2020, Introduction au numéro spécial Gérer localement la crise du Covid 19, Revue française d'administration publique, 2020/4 n 176, p.849-855.

ELIOT E., LUCAS-GABRIELLI V., MAGENEY C., 2017, Territorialisation sanitaire et décentralisation : état des lieux et enjeux à partir du cas français, Revue francophone santé et territoire, juin 2017, p. 1-11. https://journals.openedition.org/rfst/577

FLEURET S., 2016, Questionner la territorialisation de la santé en France, in Calvez M. (ed), Santé et territoire, Rennes, PUR, pp. 23-34.

FOURNIER, C., 2014, Concevoir une maison de santé pluri-professionnelle : paradoxes et enseignements d'une innovation en actes. Sciences sociales et santé, 2(2), 67-95.

Ministère des Solidarités et de la Santé, (2019), Guide d'aide à la préparation et à la gestion des tensions hospitalières et des situations sanitaires exceptionnelles.

MONZIOLS M. et al, 2020, Trois médecins généralistes sur quatre ont mis en place la téléconsultation depuis le début de l'épidémie de Covid-19, Etudes et Résultats, septembre 2020, n 1162.

PIERSON P., 1993, When Effects Become Cause. Policy Feedback and Political Change, World Politics, 45(4), p. 595-628. 
PRIBILE P., 2020, Sans les ARS, la coordination des différents acteurs du système de santé aurait été infernale (entretien), Revue française d'administration publique, 2020/4 n 176, p. 913-920.

RAMOND-ROQUIN A., ALLORY E., FIQUET L., 2020, « La concertation pluriprofessionnelle au sein des maisons de santé pluriprofessionnelles : pratiques hétérogènes et stratégies locales ", Revue française des affaires sociales, $\mathrm{n}^{\circ} 1, \mathrm{p} .125-141$.

Réseau ACCORD, 2020, Enquête nationale Maisons de Santé et Centres de Santé face au Covid-19. Résultats des deux vagues d'enquête. Rapport final, p. 1-30.

SCHWEYER F-X., 2004, « Les territoires de santé et la médecine libérale. Les enjeux d'une convergence ", Lien social et Politiques -RIAC, 52, p. 35-46.

SCHWEYER F-X., VEZINAT N., 2019, Écologie des maisons de santé pluri-professionnelles : une gouvernance multi-niveaux, Journal de gestion et d'économie de la santé, 37/1 : 3-10.

UNCAM (Union nationale des caisses d'assurance maladie). (2017). Accord Conventionnel Interprofessionnel Annexe 3, [en ligne] https://www.ameli.fr/sites/default/files/Documents/ 400408/document/texte_signe_vf.pdf. Consulté le 13/12/2020.

\section{NOTES}

1. L'article L6323-3 du Code de Santé publique définit la maison de santé pluriprofessionnelle comme une personne morale constituée entre des professionnels médicaux, auxiliaires médicaux ou pharmaciens.

2. Ces équipes de MSP doivent être organisées en Société Interprofessionnelles de Soins Ambulatoires (SISA) pour recevoir collectivement des financements permis grâce à un accord conventionnel interprofessionnel (UNCAM, 2017).

3. Ce projet bénéficie d'un financement de l'institut de recherche en santé publique (IReSP) et porte sur 10 MSP dans 4 régions. Les résultats présentés ici ne portent que sur la moitié d'entre elles pour des raisons de faisabilité en temps de pandémie.

4. Il s'agit de stages ambulatoires de 5 mois où les 3 internes ont exercé une activité clinique auprès de médecins généralistes maîtres de stage universitaires exerçant au sein des MSP étudiées et où ils ont consacré une partie de leur temps à des travaux de recherche au sein des départements de médecine générale d'Angers et Rennes. Leurs lieux de stage avaient été déterminés pour étudier 5 des 10 ESP engagées dans le programme avant le début de la crise sanitaire.

5. Groupement hospitalier de territoire, Communauté professionnelle territoriale de santé, Maison de santé pluri-professionnelle, Equipe de soins primaires coordonnée localement autour du patient.

6. CPTS communauté professionnelle territoriale de santé, elle a pour rôle de coordonner les professionnels de santé d'un même territoire, à leur initiative, qui souhaitent s'organiser autour d'un projet de santé commun.

7. Les médecins généralistes sont organisés en Association départementale pour l'organisation de la permanence des soins (ADOPS): association de médecins généralistes, responsable de l'organisation des gardes de médecine générale pour l'accès aux soins ambulatoires en dehors des heures des cabinets médicaux, après $20 \mathrm{~h}$ le soir et les week-ends. 
8. Numéro, déployé à titre expérimental dans certaines régions, pour la permanence des soins ambulatoires (soirs et week-ends). Un médecin généraliste régulateur répond aux patients, donnant des conseils ou orientant vers le médecin généraliste de garde ou vers l'hôpital. Dans certains départements, pendant la crise sanitaire, le dispositif 116117 a été également déployé sur la journée, permettant d'orienter les patients suspects de Covid-19.

9. Groupement hospitalier de territoire, fédérant les 10 établissements hospitaliers du département, dont le CHU.

10. Conseil département de l'ordre des médecins.

11. Plateforme territoriale d'appui : dispositif départemental visant à appuyer les professionnels dans l'organisation des parcours de santé. Ces structures proposent des services d'information et d'orientation des professionnels vers les différentes ressources (sanitaires, sociales et médico-sociales) du territoire, d'appui à l'organisation des parcours, et de soutien aux pratiques et initiatives professionnelles.

12. Groupement hospitalier de territoire, fédérant les 3 hôpitaux généraux et les 4 hôpitaux locaux du département

13. Monziols et al (2020) ont montré que c'était souvent le cas dans les régions les moins touchées par l'épidémie.

14. Maison des adolescents

\section{RÉSUMÉS}

Cet article propose une lecture territoriale de la pandémie de Covid-19 en analysant sa gestion par cinq équipes de soins primaires organisées en maisons de santé pluri-professionnelles (MSP) dans deux régions, les Pays de la Loire et la Bretagne. A partir de l'exploitation de résultats intermédiaires d'un programme de recherche (Concert-MSP) qui étudie les formes et modalités de concertation pluri-professionnelle dans les MSP, l'analyse combine deux dimensions territoriales : les territoires de proximité dans lesquels des équipes de MSP se sont adaptées à la situation inédite et les territoires régionaux qui ont été les espaces du déploiement des mesures de sécurité sanitaire décidées par l'Etat. La première partie présente la diversité des contextes territoriaux (territoires à tendance rurale, rural intermédiaire ou à tendance urbaine) des 5 MSP étudiées qui ont des logiques de fonctionnement variés : maintien d'une offre de soins local, logique de fonctionnement centrée sur les professionnels ou logique populationnelle. La deuxième partie, centrée sur la gestion de la crise sanitaire en soins primaires, étudie le territoire régional comme espace d'application d'un plan national, avec deux approches différentes quant à la mobilisation des acteurs locaux et de l'élaboration de nécessaires compromis à passer localement pour assoir l'autorité de l'Etat, mais aussi comment les équipes ont mis en place de nouveaux modes d'organisation. La troisième partie analyse comment les équipes des MSP ont modifié leurs pratiques de soins et de coopération pour gérer la crise. Leur mobilisation a permis l'émergence ou le renforcement de réseaux d'entraide locaux, et a interrogé les territoires professionnels et la division symbolique du travail contribuant ainsi à donner de nouveaux rôles et à instaurer de nouvelles pratiques. Si l'existence d'un collectif de 
travail a été facilitant pour élaborer des réponses appropriées, le rôle central des médecins a, lui, été plutôt renforcé. L'élargissement des réseaux relationnels et l'interconnaissance entre acteurs des territoires pourraient compter parmi les acquis de la crise.

This article offers a spatialized reading of the COVID 19 pandemic by analysing its management by five primary care teams organized in multi-professional health homes (MSPs) in two regions, the pays de la Loire and Brittany. Based on the exploitation of intermediate results of a research program (Concert-MSP) that studies the forms and modalities of multi-professional consultation in MSPs, the analysis combines two territorial dimensions: the local territories in which MSP teams have adapted to the unprecedented situation and the regional territories that have been the spaces for the deployment of health security measures decided by the State.

The results show the diversity of territorial contexts (territories with a rural, intermediate or urban trend) of the 5 MSPs studied that have resulted in varied operating situations seen as combination of territorial factors more or less favourable to the proposed coordinated exercise (focus on maintaining a local care offer, focus on professionals cooperation, focus on community). Using these categories, the article analysis the management of the health crisis in primary care, by taking the regional territory as a space for the implementation of a national plan.

Two different approaches are observed to mobilizing local actors and developing necessary compromises to be made locally to establish state authority. Basically, the regional health agency in Pays de la Loire favoured an approach where patients suspected of COVID-19 infection were referred to dedicated places, separated from the usual places of care, to provide medical consultations and, in a second stage, diagnostic tests. The Brittany agency, for its part, has deployed an approach based on existing organizations after listing them, and carried out followup work. Medical teams have set up new organizations in relation with these differentiated regional strategies but also in relation with local specificities. The analysis of the activities of health professionals within the framework of MSPs mobilizes a new definition of the notion of territory, of a sociological nature. This is professional territory, in which each professional group works to define a specific perimeter of intervention bounded by borders that sometimes impede cooperation (Schweyer, 2004). The Covid crisis has shaken up these territories by renewing the issue of patient care. Within each team, a double question arose: who supports what? With whom? Indeed, MSPs teams have changed their care and cooperation practices to manage the crisis. Their mobilization enabled the emergence or strengthening of local self-help networks, and questionned the professional territories and the symbolic division of labour, thus helping to give new roles and establish new practices. While the existence of a working collective has been facilitated in developing appropriate responses, the central role of physicians has been rather strengthened. The expansion of relational networks and the inter-knowledge between actors in the territories could be among the achievements of the crisis. But the drawing of territories do not depend only on professions, they are negotiated with the state, as recalled by the management of the crisis where certain professions have been prevented from practising in liberal practice (speech therapists, dieticians, podiatrists, physiotherapists, etc.). For those who had a mixed liberal-hospital activity, they increased the time in structure. The physiotherapists experienced a forced withdrawal from their practice on the advice of the College Board, with instructions to maintain urgent care at home (which definition has sometimes been discussed within the teams). Some called patients for advice and posted videos on Youtube. Some salaried professionals (ex. public health nurse) have ceased their activity within the MSP at the request of their employer. Other professionals have been given new roles. Pharmacists, for example, have had new missions given by the state such as identifying domestic violence.

The day-to-day activity of the teams has been substantially modified by the priority given to the management of the epidemic. Activities such as multi-professional consultation, have been 
abandoned or adapted to the context of the crisis. It should be remembered that the two regions involved in the analysis were relatively spared from the epidemic. Not all elements of the crisis management system have been deployed and the teams mobilized to receive infected patients have been moderately exposed. This lesser pressure has no doubt fostered a space for local initiatives to respond to the crisis. However, the territorial differentiation observed in the management of the crisis can be explained in large part in the configuration of the territory of each MSP. For example, MSP 1 backed by a proximity hospital was able to manage the crisis on its own, which was impossible for MSP 4 both close to a "leading" hospital and whose population served faces more difficulties in access to care. MSP 3 away from a hospital and in conjunction with an ARS departmental delegation encouraging local initiatives was able to manage the Covid system fairly autonomously.

\section{AUTEURS}

\section{FRANÇOIS-XAVIER SCHWEYER}

Sociologue, Professeur de l'Ecole des Hautes Etudes en Santé Publique, Centre Maurice Halbwachs UMR 8097

\section{LAURE FIQUET}

MD, 1 - Univ Rennes, Département de médecine générale, 2 - CHU Rennes, Inserm, CIC 1414 [(Centre d'Investigation Clinique de Rennes)], F-35000 Rennes, France.

\section{SÉBASTIEN FLEURET}

Géographe, DR CNRS, ESO - UMR 6590

\section{MARIE BLOIS}

Interne, Département de médecine générale, UFR Santé, Univ d'Angers, France

\section{MARIE GARNIER}

Interne, Département de médecine générale, UFR Santé, Univ d'Angers, France

\section{ANTOINE GÉAL}

Interne, Univ Rennes, Département de Médecine Générale, F-35000 Rennes, France

\section{ALINE RAMOND-ROQUIN}

MD, PhD, 1 - MCU, Département de médecine générale, UFR Santé, Univ d'Angers, France et 2 Irset (Institut de recherche en santé, environnement et travail) - UMR_S 1085, Université d'Angers, Université de Rennes, Inserm, EHESP, F-49000 Angers, France 